'Departamento de Medicina

Interna, Escuela de Medicina, Universidad de Valparaíso. Valparaíso, Chile.

${ }^{2}$ Centro de Investigaciones Biomédicas, Escuela de Medicina, Universidad de Valparaíso. Valparaíso, Chile.

${ }^{3}$ Servicio de Cardiología, Clínica Bupa Reñaca. Viña del Mar, Chile. ${ }^{4}$ Unidad de Cuidados Intensivos, Clínica Bupa Reñaca . Viña del Mar, Chile.

${ }^{5}$ Departamento de Estadística, Facultad de Ciencias, Universidad de Valparaíso. Valparaíso, Chile. aPhD.

Trabajo no recibió financiamiento. Los autores declaran no tener conflictos de interés.

Recibido e 18 de julio de 2020, aceptado el 28 septiembre de

Correspondencia a: Dr. Rienzi Díaz Navarro Departamento de Medicina Interna, Universidad de Valparaíso, Calle Angamos 655,

Viña del Mar, Chile. diaz.rienzi@gmail.com

\section{Valor de la resonancia magnética cardíaca para el diagnóstico diferencial en pacientes con sospecha de infarto agudo de miocardio con elevación del segmento ST y arterias coronarias normales}

\author{
RIENZI DÍAZ-NAVARRO ${ }^{1,2,3}$, DANILO SILVA GONZÁLEZ ${ }^{4}$, \\ CARLOS HENRÍQUEZ-ROLDÁN ${ }^{5, a}$
}

\section{Cardiac magnetic resonance imaging in patients with a suspected myocardial infarction and normal coronary arteries}

Background: Patients hospitalized with suspected ST-segment elevation myocardial infarction (STEMI) who have normal coronary arteries (CAs) on invasive coronary angiography (ICA) may have an AMI or another acute cardiac disease that mimics it. Aim: To evaluate the usefulness of cardiac magnetic resonance imaging (CMRI) for diagnosing conditions resembling AMI with normal CAs. Material and Methods: We studied 424 consecutive patients admitted with suspected STEMI who underwent ICA. Those with normal CAs underwent CMRI involving cine-CMRI sequences to evaluate segmental wall motion, T2-weighted short-tau inversion-recovery imaging to detect oedema and delayed contrast enhancement (DCE) after gadolinium administration to identify necrosis/fibrosis. Patients with previous myocardial infarction were excluded. Results: Twenty-six patients (6.1\%) had normal CAs. Definitive diagnosis after CMRI was acute myocarditis in 11 patients (42.3\%) whose DCE was localized in the subepicardium or intramyocardially but not in the endocardium, AMI in nine patients (34.6\%) who had subendocardial or transmural DCE, and Takotsubo cardiomyopathy (TCM) in six patients (23.1\%), whose CMRI showed regional contractility abnormalities of the left ventricle and myocardial oedema but not DCE. Conclusions: Cardiac magnetic resonance imaging allows a precise diagnosis of acute myocardial infarction in patients with angiographically normal coronary arteries.

(Rev Med Chile 2020; 148: 1406-1417)

Key words: Magnetic Resonance Imaging; Myocarditis; ST Elevation Myocardial Infarction; Takotsubo Cardiomyopathy.

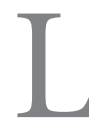

os pacientes hospitalizados por sospecha de infarto agudo de miocardio con elevación del segmento ST (IAM con SDST) que tienen arterias coronarias normales en la coronariografía posterior a su admisión al hospital, pueden tener un infarto agudo de miocardio (IAM) u otra una afección cardíaca aguda que lo simule ${ }^{1-4}$. En este escenario, el diagnóstico diferencial representa un desafío y es esencial para efectuar un tratamiento correcto. El diagnóstico de IAM se plantea en 
presencia de dolor torácico prolongado de carácter anginoso acompañado de cambios electrocardiográficos isquémicos y elevación de la troponina plasmática $^{5}$. La resonancia magnética cardíaca (RMC) es un método de diagnóstico no invasivo de alta sensibilidad tanto para el diagnóstico de la patología cardíaca aguda de orígen isquémico como no isquémico ${ }^{6-9}$.

El objetivo principal de este estudio fue evaluar la utilidad de la RMC en el diagnóstico diferencial de pacientes hospitalizados con diagnóstico de IAM con SDST cuyo árbol coronario resultó normal en la coronariografía.

\section{Material y Métodos}

Este estudio incluyó 424 pacientes hospitalizados consecutivamente con sospecha de IAM con SDST en la Unidad de Cuidados Intensivos de la Clínica Reñaca, Viña del Mar. Todos los pacientes ingresaron con dolor torácico opresivo prolongado de reposo ( $>30 \mathrm{~min}$ ), alteraciones electrocardiográficas con supradesnivel del segmento ST $>0,2$ $\mathrm{mV}$ en dos o más derivaciones precordiales contiguas y/o $>0,1 \mathrm{mV}$ en otras derivaciones y elevación de la troponina plasmática. En todos los pacientes se realizó un ecocardiograma bidimensional transtorácico antes de la coronariografía, para pesquisar alteraciones segmentarias de la contracción y estimar la función global del ventrículo izquierdo. En 26 pacientes sin lesiones ateroescleróticas en la coronariografía se realizó RMC tres a cuatro días después de la coronariografía. Los pacientes con infarto al miocardio previo, lesiones coronarias obstructivas en la coronariografía, otras enfermedades cardíacas conocidas o contraindicaciones para realizar una RMC fueron excluidos. El Comité de Ética local aprobó este estudio y todos los pacientes firmaron el consentimiento informado autorizando su participación en el estudio.

\section{Estudios de RMC}

Los estudios se realizaron de manera protocolizada en un resonador SIGNA Excite 1.5-Tesla (General Electric Medical Systems, Milwaukee, $\mathrm{MN}, \mathrm{EE}$. UU.). Las imágenes se adquirieron mediante una antena de superficie corporal acoplada en fase durante apneas repetidas y utilizando sincronización electrocardiográfica. Las imágenes de cine-resonancia se adquirieron utilizando secuencias de precesión libre en estado estacionario, en proyecciones de dos, tres, cuatro cavidades y en proyecciones de eje corto desde la base hasta el ápex, para evaluar la contracción segmentaria y global del corazón ${ }^{10}$. Para detectar edema miocárdico se utilizaron secuencias segmentadas T2STIR (short tau inversion recovery), realizándose análisis de la intensidad de señal del miocardio normalizada al músculo esquelético mediante el programa CV42 (Version 5.12.1, Circle Cardiovascular Imaging Inc., Calgary, Canada). El azul indica una relación de intensidad de señal de miocardio/ músculo esquelético $\geq 2.0$ que indica edema y el color verde indica una intensidad de señal normal (1.4-1.9) (Figura 1) ${ }^{11}$. Para la identificación de necrosis y fibrosis, se administró $0,2 \mathrm{mmol} / \mathrm{kg}$ de gadolinio endovenoso con adquisición de las imágenes 10 minutos después, utilizándose secuencias T1 modificadas con la aplicación de un pulso de inversión de $180^{\circ}$, para anular la señal del miocardio sano que se visualiza de color negro, mientras que el miocardio con necrosis o fibrosis aparece con realce tardío ${ }^{11,12}$. Las secuencias T2-STIR y de realce tardío fueron adquiridas en proyecciones idénticas a las del cine resonancia.

Se diagnosticó IAM en pacientes con áreas de realce tardío subendocárdico y transmural; miocarditis aguda en aquellos con la misma alteración, pero de localización subepicárdica o intramiocárdica y miocardiopatía de Takotsubo en pacientes con edema miocárdico y ausencia de realce tardío. Los estudios de RCM fueron evaluados por dos médicos expertos en esta técnica de diagnóstico sin conocimiento de los datos clínicos de los pacientes.

\section{Estadística}

Para la realización de los análisis estadísticos se utilizó Stata (versión 16, StataCorp, College Station, TX, EE. UU.). Los datos continuos se expresaron como medias (DE). Se compararon los hallazgos clínicos, de laboratorio, ecocardiográficos y de RMC entre subgrupos usando análisis de varianza. Para variables categóricas se usó el test de chi-cuadrado, correlación de Pearson y el test exacto de Fisher. Todas las pruebas fueron de dos colas. Un valor de $\mathrm{p}<0.05$ se consideró como significativo.

\section{Resultados}

De los 424 pacientes hospitalizados con diagnóstico presuntivo de IAM con SDST, 26 pacientes 


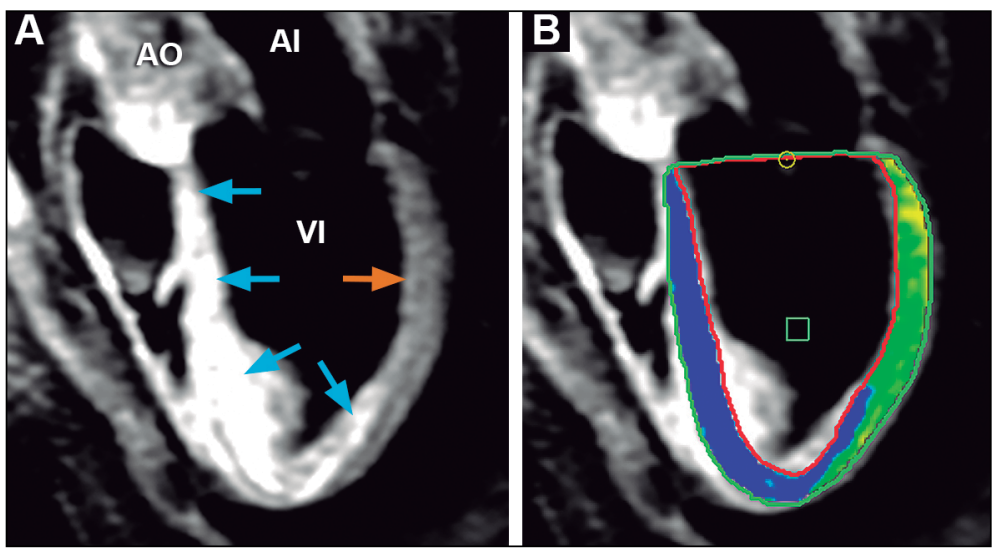

Figura 1. A) Resonancia magnética cardíaca con imagen de secuencia T2-STIR en visión de tres cavidades que muestra un aumento de la intensidad de la señal (Flechas celestes) en la región anteroseptal y apical del VI en comparación al miocardio normal (Flecha naranja) indicando la existencia de edema miocárdico. B) Análisis de la intensidad de señal de la imagen ponderada en T2 mediante el uso del programa CVI42 (ver métodos) con visualización codificada por colores de la intensidad de la señal relativa normalizada al músculo esquelético. El azul indica una relación de intensidad de señal de miocardio/músculo esquelético $\geq 2,0$ que indica edema y el color verde indica una intensidad de señal normal $(1,4-1,9)$. El borde endocárdico está representado por la línea roja y el borde epicárdico por la línea verde. Al, aurícula izquierda; AO, aorta; VI, ventrículo izquierdo.

$(6,1 \%)$ tenían arterias coronarias sin lesiones ateroescleróticas en la coronariografía. La edad de los pacientes fue 47,7 $\pm 16,3$ años y la concentración máxima de troponina $24,4 \pm 37,0 \mathrm{ng} / \mathrm{ml}$. En la Tabla 1, se muestran las características clínicas basales y los hallazgos de laboratorio. El diagnóstico definitivo luego de la RMC fue miocarditis aguda en 11 pacientes (42,3\%), todos con áreas de realce tardío subepicárdico o intramiocárdico sin compromiso del endocardio acompañado de edema miocárdico (Figura 2); IAM en nueve pacientes $(34,6 \%)$, todos con áreas de realce tardío subendocárdico o transmural y edema miocárdico (Figura 3); y miocardiopatía de Takotsubo en seis

Tabla 1. Antecedentes clínicos y de laboratorio

\begin{tabular}{|c|c|c|c|c|}
\hline & $\begin{array}{l}\text { Miocarditis aguda } \\
\qquad(n=11)\end{array}$ & $\begin{array}{c}\text { IAM } \\
(n=9)\end{array}$ & $\begin{array}{c}\text { MCT } \\
(n=6)\end{array}$ & Valor de $\mathbf{P}$ \\
\hline Edad (años) & $35,8 \pm 11,8$ & $48,4 \pm 9,5^{\ddagger}$ & $68,3 \pm 8,5^{\varnothing}$ & $\begin{array}{l}<0,05^{\ddagger} \\
<0,001^{\varnothing}\end{array}$ \\
\hline Hipertensión & 2 of 11 & 7 of $9^{\#}$ & 3 of 6 & $<0,001^{\#}$ \\
\hline Dislipidemia & 2 of 11 & 4 of 9 & 1 of 6 & NS \\
\hline Tabaquismo & 7 of 11 & 6 of 9 & 2 of 6 & NS \\
\hline Diabetes Mellitus & 0 of 11 & 2 of 9 & 1 of 6 & NS \\
\hline Troponina I (g/mL) & $17,9 \pm 19,4$ & $32,7 \pm 32,9^{\$}$ & $4,0 \pm 2,1$ & $<0,05^{\$}$ \\
\hline CK-MB (ng/mL) & $54,0 \pm 54,8$ & $95,6 \pm 97,6^{¥}$ & $10,7 \pm 6,1$ & $<0,05^{¥}$ \\
\hline PNC (pg/mL) & $125,7 \pm 102,1$ & $121,1 \pm 143,6$ & $547,8 \pm 44,3^{8}$ & $<0,005^{\&}$ \\
\hline PCR (mg/L) & $41,4 \pm 22,9^{\pi}$ & $7,8 \pm 7,5$ & $3,9 \pm 4,2$ & $<0,0001^{\pi}$ \\
\hline Leucocitos $\left(\times 10^{6} / \mu \mathrm{L}\right)$ & $8,8 \pm 2,9$ & $11,3 \pm 4,9$ & $9,5 \pm 4,9$ & NS \\
\hline
\end{tabular}

IAM: infarto agudo de miocardio; MCT: miocardiopatía de Takotsubo; ${ }^{\prime} \mid \mathrm{AM}$ versus miocarditis aguda; ${ }^{\varnothing} \mathrm{MCT}$ versus IAM y miocarditis aguda; " $I$ IAM versus myocarditis aguda y MCT; ${ }^{~ I} \mathrm{AMM}$ versus $\mathrm{MCT} ;{ }^{*} \mathrm{IAM}$ versus $\mathrm{MCT} ;{ }^{\circledR} \mathrm{MCT}$ versus IAM y miocarditis aguda; "miocarditis aguda versus IAM y MCT; CK-MB: creatinquinasa MB; PNC: péptido natriurético cerebral; PCR: proteína C reactiva. 

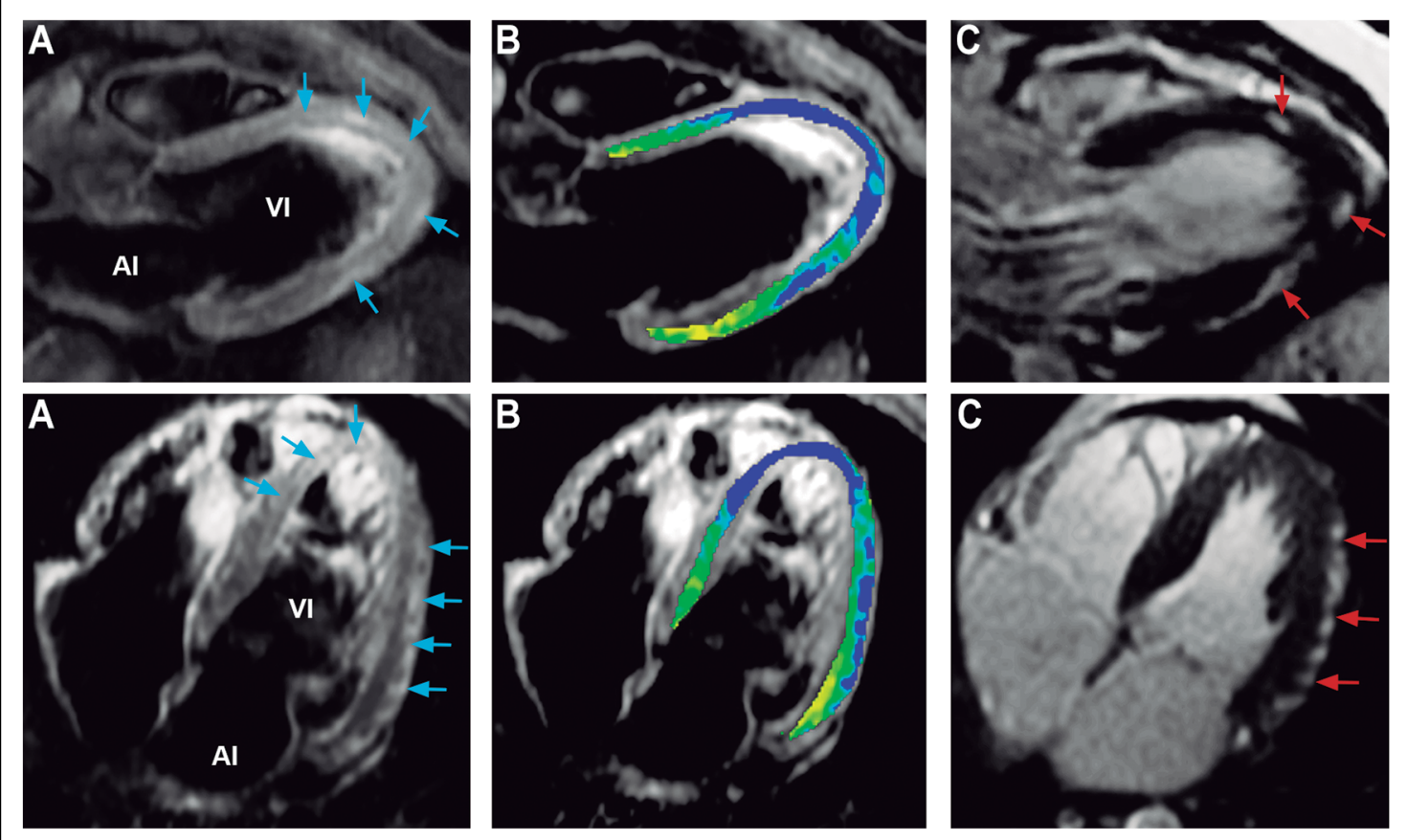

Figura 2. Resonancia magnética cardíaca en dos pacientes con miocarditis aguda. Arriba: visión de tres cavidades. A) Imagen con secuencia T2-STIR que muestra edema subepicárdico e intramiocárdico con distribución parcheada (Flechas celestes) en los segmentos anteroapical e inferolateral medial y apical del ventrículo izquierdo (VI). B) Análisis de la intensidad de señal de la imagen ponderada en T2 mediante el uso del programa CVI42 (ver métodos) con visualización codificada por colores de la intensidad de la señal relativa, normalizada al músculo esquelético. El azul indica una relación de intensidad de señal de miocardio/ músculo esquelético $\geq 2,0$ que indica edema y el color verde indica una intensidad de señal normal $(1,4-1,9)$. C) Imagen con secuencia de realce tardío de gadolinio en la que se identifican focos de hiperrealce de gadolinio de localización subepicárdica e intramural en el área de miocardio con edema (Flechas rojas). Abajo: visión de cuatro cavidades. A) Imagen con secuencia T2-STIR en la que se observa edema subepicárdico en la pared lateral del VI (Flechas celestes). B) Análisis de la intensidad de señal de la imagen ponderada en T2 mediante el uso del programa CVI42 previamente descrito confirmando la localización del edema. C) Imagen con secuencia de realce tardío de gadolinio que identifica hiperrealce subepicárdico localizado en la pared lateral del VI en el área de miocardio con edema (Flechas rojas). Al, aurícula izquierda.

pacientes $(23,1 \%)$, todos con edema miocárdico y ausencia de áreas de realce tardío (Figura 4).

La mayoría de los pacientes con IAM o miocarditis aguda fueron hombres, en cambio, los con miocardiopatía de Takotsubo fueron todas mujeres. La edad promedio de los pacientes con miocarditis aguda fue menor a los enfermos con IAM o miocardiopatía de Takotsubo $(35,8,48,4$ y 68,3 años, respectivamente); $\mathrm{p}<0,05)$. Los valores máximos de troponina y creatinquinasa MB fueron más altos en los pacientes con IAM que los pacientes con miocardiopatía de Takotsubo $(32,7 \mathrm{ng} / \mathrm{ml}$ versus $4,0 \mathrm{ng} / \mathrm{mL}$ y $95,6 \mathrm{ng} / \mathrm{mL}$ versus $10,7 \mathrm{ng} / \mathrm{ml}$, respectivamente; $\mathrm{p}<0,05)$. Por el contrario, los valores máximos de proteí- na $C$ reactiva fueron superiores en los pacientes con miocarditis aguda respecto a los con IAM o miocardiopatía de Takotsubo $(41,4 \mathrm{mg} / \mathrm{L}, 7,8$ $\mathrm{mg} / \mathrm{L}$ y $3,9 \mathrm{mg} / \mathrm{L}$, respectivamente; $\mathrm{p}<0,0001)$. El péptido natriurético cerebral máximo fue mayor en los pacientes con miocardiopatía de Takotsubo que en los pacientes con IAM o miocarditis aguda $(547,8 \mathrm{pg} / \mathrm{mL}, 121.1 \mathrm{pg} / \mathrm{mL}$ y $125,7 \mathrm{pg} /$ $\mathrm{mL}$, respectivamente; $\mathrm{p}<0,005)$. En la Tabla 2, se presentan los hallazgos de la E2D y RMC. La fracción de eyección del VI estimada por ambas técnicas fue menor en los pacientes con miocardiopatía de Takotsubo que en los pacientes con miocarditis aguda o IAM ( $\mathrm{p}<0,005)$. Todos los pacientes con IAM o miocardiopatía de Takotsubo 

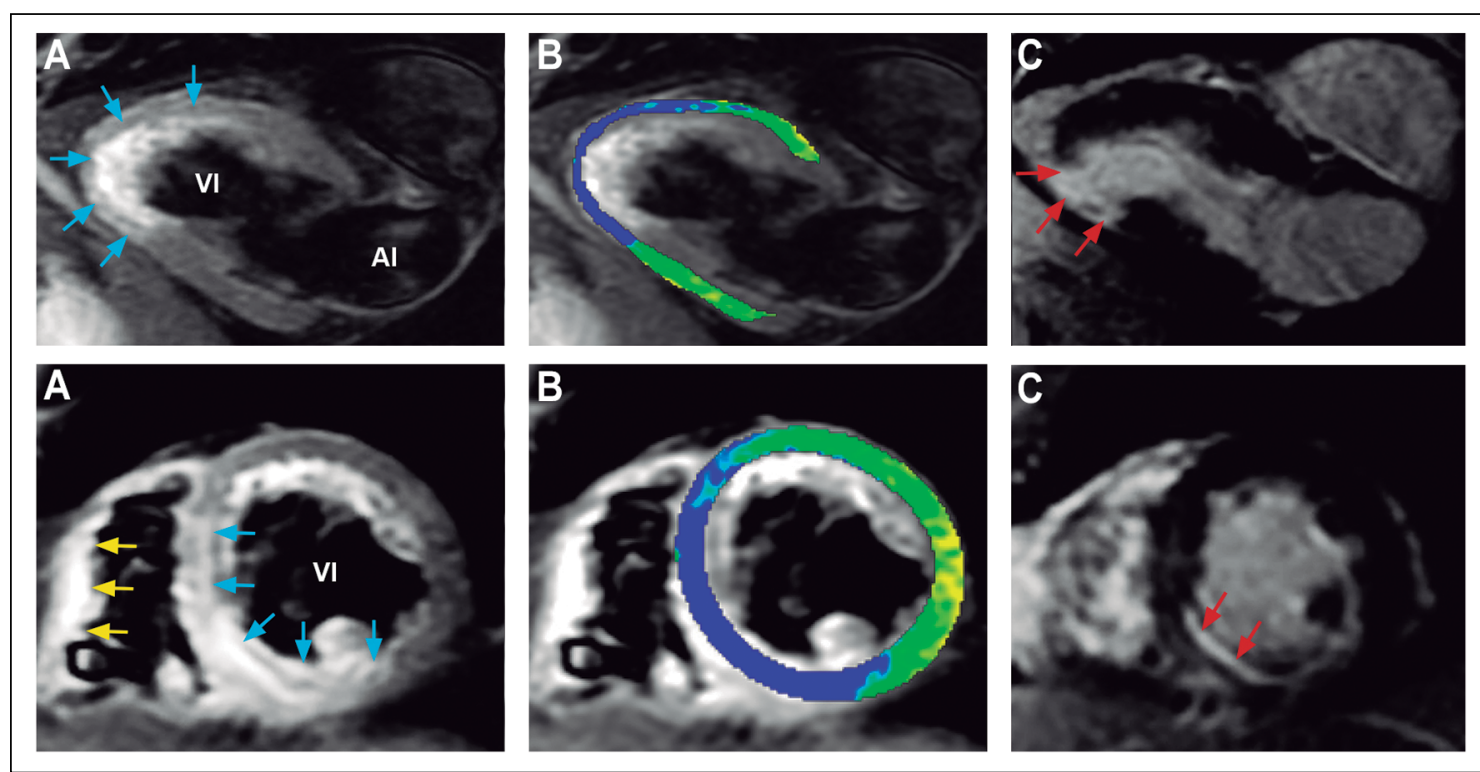

Figura 3. Resonancia magnética cardíaca en dos pacientes con infarto agudo de miocardio. Arriba: A) Imagen de dos cavidades con secuencia T2-STIR que muestra edema subepicárdico en segmentos inferoapical y anteroapical del ventrículo izquierdo (VI) (Flechas celestes). B) Análisis de la intensidad de señal de la imagen ponderada en T2 mediante el uso del programa CVI42 (ver métodos) con visualización codificada por colores de la intensidad de la señal relativa, normalizada al músculo esquelético. El azul indica una relación de intensidad de señal de miocardio/músculo esquelético $\geq 2,0$ que indica edema y el color verde indica una intensidad de señal normal $(1,4-1,9)$. C) Imagen con secuencia de realce tardío de gadolinio en la que se identifica hiperrealce inferoapical transmural (Flechas rojas), cuyo tamaño es menor al área de miocardio con edema. Abajo: A) Imagen con secuencia T2-STIR en eje corto del ventrículo izquierdo a nivel de los músculos papilares que muestra edema en la pared inferior y septo interventricular (Flechas celestes), además, se identifica edema en la pared lateral del ventrículo derecho (Flechas amarillas). B) Análisis de la intensidad de señal de la imagen ponderada en T2 mediante el uso del programa CVI42 previamente descrito confirmando la localización del edema. C) imagen con secuencia de realce tardío de gadolinio que muestra hiperrealce subendocárdico en la pared inferior y una pequeña porción del septo inferior, cuyo tamaño es claramente menor al área de miocardio con edema (Flechas rojas). Al, aurícula izquierda.

tenían alteraciones segmentarias de la contracción del VI cuya localización era concordante entre la ecocardiografía y la cine-resonancia (Figura 5). En la mayoría de los pacientes se identificó derrame pericárdico laminar leve, independientemente del diagnóstico y sin diferencias entre las 3 patologías.

En los pacientes con miocarditis aguda, el edema miocárdico y las áreas de realce tardío estaban localizadas principalmente en la pared lateral del VI en la mayoría de los casos (Figura 2). En los enfermos con IAM, 4 pacientes presentaban realce tardío subendocárdico y 5 transmural (Figura 3). En todos se identificó edema miocárdico localizado en el área de necrosis y en algunos pacientes este se extendía más allá del territorio infartado (Figura 3). De las pacientes con miocardiopatía de Takotsubo, 4 de ellas tenían acinesia ventricular izquierda apical y circunferencial medial con hipercontractilidad basal y 2 tenían acinesia ventricular izquierda medial con hipercontractilidad basal y apical (Figura 4). En todos los pacientes se observó edema miocárdico localizado en la región discinética sin identificarse áreas de realce tardío (Figura 5). La ecocardiografía de control mostró regresión de las alteraciones segmentarias de la contractilidad y normalización de la función global del VI en todos los pacientes con miocardiopatía de Takotsubo (Figura 6).

\section{Discusión}

Este es el primer estudio nacional que muestra la utilidad de la RMC en el diagnóstico diferencial de pacientes hospitalizados con sospecha diagnóstica de IAM con SDST, en los que se encontró un 

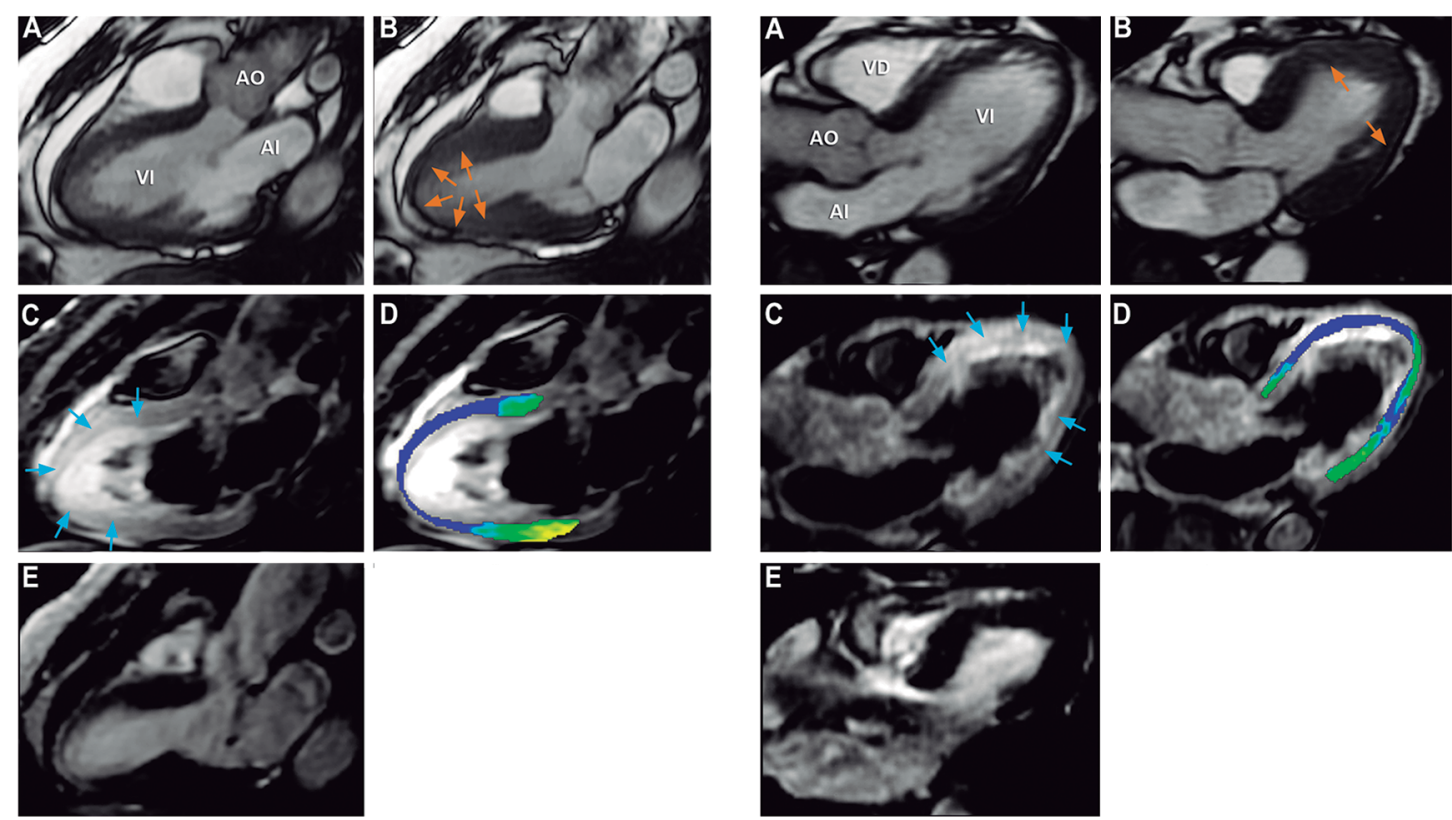

Figura 4. Izquierda: resonancia magnética cardíaca visión de tres cavidades en una paciente con miocardiopatía de Takotsubo típica. A y B) imágenes de cine resonancia cardíaca en diástole y sístole, respectivamente. En sístole se observa acinesia apical y medial del ventrículo izquierdo. C) secuencia T2-STIR con edema localizado en la región acinética (Flechas celestes). D) Análisis de la intensidad de señal de la imagen ponderada en T2 mediante el uso del programa CVI42 (ver métodos) con visualización codificada por colores de la intensidad de la señal relativa, normalizada al músculo esquelético. El azul indica una relación de intensidad de señal de miocardio/músculo esquelético $\geq 2,0$ que indica edema y el color verde indica una intensidad de señal normal $(1,4-1,9)$. E) secuencia de realce tardío con ausencia de hiperrealce. Derecha: resonancia magnética cardíaca visión de tres cavidades en una paciente con miocardiopatía de Takotsubo atípica. A y B) imágenes de cine resonancia en diástole y sístole respectivamente. En sístole se observa acinesia medial del ventrículo izquierdo (Flecha naranjas). C) secuencia T2-STIR con edema localizado en la región acinética que se extiende hacia el segmento anteroapical (Flechas celestes). D) Análisis de la intensidad de señal de la imagen ponderada en T2 mediante el uso del programa CVI42 previamente descrito. E) secuencia de realce tardío con ausencia de hiperrealce. Al, aurícula izquierda, AO, aorta; VI, ventrículo izquierdo; VD, ventrículo derecho.

Tabla 2. Hallazgos en la ecocardiografía y resonancia magnética cardíaca

\begin{tabular}{|c|c|c|c|c|}
\hline & $\begin{array}{l}\text { Miocarditis aguda } \\
\qquad(n=11)\end{array}$ & $\begin{array}{c}\text { IAM } \\
(n=9)\end{array}$ & $\begin{array}{c}\text { MCT } \\
(n=6)\end{array}$ & Valor de $\mathbf{P}$ \\
\hline \multicolumn{5}{|l|}{ Ecocardiograma 2D } \\
\hline Derrame pericárdico & 8 de 11 & 3 de 9 & 2 de 6 & NS \\
\hline ASCVI & 0 de $11^{\S}$ & 9 de 9 & 6 de 6 & $<0,001^{\S}$ \\
\hline FEVI (\%) & $61,9 \pm 1,6$ & $60,1 \pm 4,9$ & $54,3 \pm 4,7^{8}$ & $<0,005^{\&}$ \\
\hline \multicolumn{5}{|l|}{ Resonancia cardiaca } \\
\hline VFDVli $\left(\mathrm{ml} / \mathrm{m}^{2}\right)$ & $73,9 \pm 9,4$ & $66,8 \pm 19,2$ & $58,4 \pm 12,6$ & NS \\
\hline VFSVli $\left(\mathrm{ml} / \mathrm{m}^{2}\right)$ & $30,0 \pm 5,7$ & $28,6 \pm 8,7$ & $27,1 \pm 9,8$ & NS \\
\hline FEVI (\%) & $61,5 \pm 3,8$ & $60,1 \pm 4,9$ & $51,2 \pm 4,8^{7}$ & $<0,005^{*}$ \\
\hline MVli $\left(\mathrm{g} / \mathrm{m}^{2}\right)$ & $66,0 \pm 12,0$ & $59,1 \pm 9,3$ & $27,1 \pm 9,8$ & NS \\
\hline Derrame pericárdico & 10 de 11 & 6 de 9 & 4 de 6 & NS \\
\hline
\end{tabular}

IAM: infarto agudo de miocardio; MCT: miocardiopatía de Takotsubo; §miocarditis aguda versus IAM y MCT; ${ }^{\circledR}$ MCT versus IAM y miocarditis aguda; ${ }^{\ddagger} \mathrm{MCT}$ versus IAM y miocarditis aguda; ASCVI, alteración segmentaria de la contracción ventrícular izquierda; FEVI, fracción de eyección del ventrículo izquierdo; VFDVIi, volumen de fin de diástole del ventrículo izquierdo indexado; VFSVli, volumen de fin de sístole del ventrículo izquierdo indexado; MVli, masa del ventrículo izquierdo indexada. 

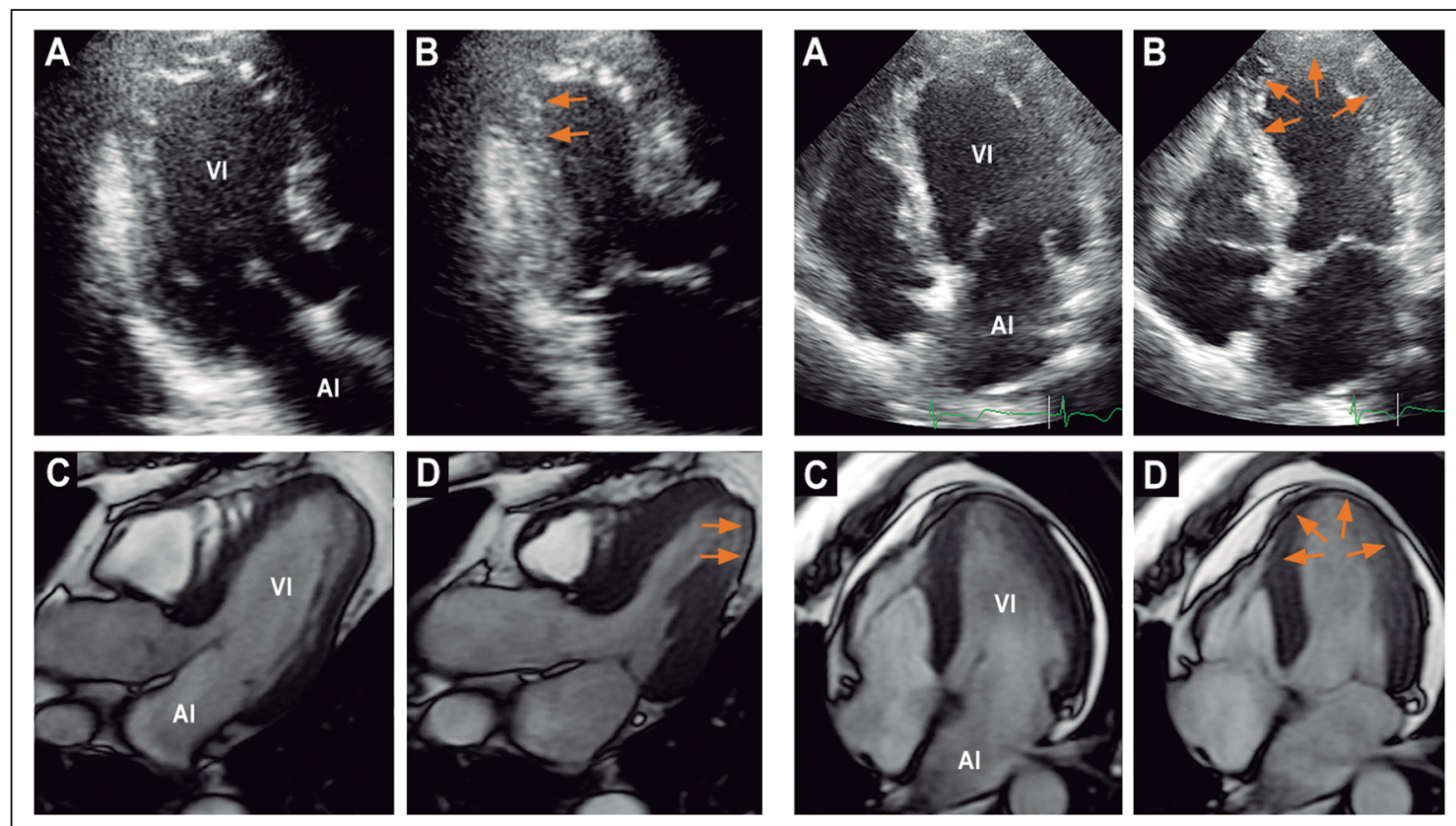

Figura 5. Izquierda arriba: ecocardiograma bidimensional, visión de tres cavidades en un paciente con infarto agudo de miocardio. A y B) imágenes en diástole y sístole, respectivamente. En sístole se observa acinesia inferoapical (Flechas naranjas). Izquierda abajo: cine resonancia cardíaca, visión de tres cavidades. C y D) imágenes en diástole y sístole, respectivamente. En sístole se identifica acinesia inferoapical que coincide con lo observado en la ecocardiografía (Flechas naranjas). Derecha arriba. ecocardiograma bidimensional visión de 4 cavidades en una paciente con miocardiopatía de Takotsubo típica. A y B) imágenes en diástole y sístole, respectivamente. En sístole se observa acinesia apical y medial del ventrículo izquierdo (Flechas naranjas). Derecha abajo: cine resonancia cardíaca, visión de cuatro cavidades. C y D) imágenes en diástole y sístole, respectivamente. En sístole se identifica área discinética similar a la observada en el ecocardiograma (Flechas naranjas). Al, aurícula iquierda, VI, ventrículo izquierdo.

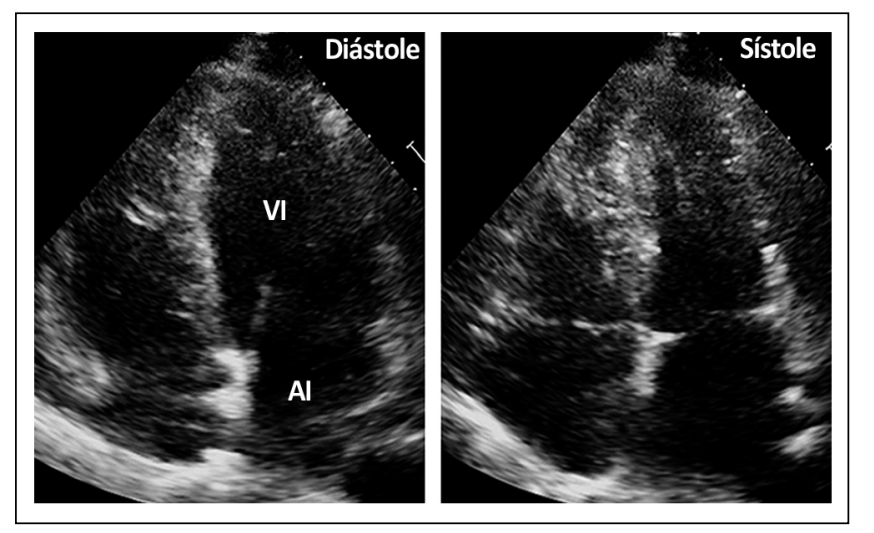

Figura 6. Imagen de 4-cavidades de un ecocardiograma bidimensional de control en una paciente con miocardiopatía de Takotsubo típica cuyo ecocardiograma de ingreso se muestra en la Figura 5 arriba derecha. En la imagen sistólica, hay completa regresión del trastorno de contracción que se observa en la Figura 5B. Al, aurícula izquierda, VI, ventrículo izquierdo. árbol coronario sin lesiones ateroescleróticas en la coronariografía.

Los criterios universales para el diagnóstico de IAM con SDST son dolor torácico prolongado de carácter anginoso, alteración electrocardiográfica con supradesnivel del segmento ST y elevación de la troponina plasmática ${ }^{5}$. Sin embargo, algunos pacientes con las características descritas no tienen un IAM y representan falsos positivos ${ }^{14-19}$. Otros pacientes tienen enfermedades cardíacas 
agudas de orígen no isquémico que imitan al IAM $^{6-9,14-17}$. En pacientes hospitalizados con sospecha de IAM con SDST, aproximadamente 2,3-14\% tienen arterias coronarias normales en la coronariografía $^{14-19}$, información concordante con $6,1 \%$ observado en esta cohorte. En la actualidad, la RMC es una herramienta diagnóstica útil para confirmar el diagnóstico de IAM o diagnosticar otra patología cardíaca aguda no isquémica que lo imite, en pacientes hospitalizados con diagnóstico de IAM con SDST y cuyo árbol coronario es normal en la coronariografía ${ }^{6-9}$. En estos pacientes, la RMC permite modificar el diagnóstico de ingreso y consecuentemente cambiar la estrategia terapéutica ${ }^{20}$. Los pacientes en los que se confirma un IAM corresponden al cuadro clínico conocido por sus siglas en inglés como MINOCA (Myocardial Infarction With no Obstructive Coronary Arteries $)^{21-24}$. La RCM permite la identificación de edema miocárdico (Figuras 2 y 3) 18,19,25,26 y áreas de necrosis o fibrosis después de la administración de gadolinio (Figuras 2 y 3$)^{27,28}$. En este estudio, nueve $(34,6 \%)$ de los 26 pacientes con arterias coronarias normales tenían un IAM, identificándose en todos áreas de realce tardío subendocárdico o transmural, patrón que es característico de daño miocárdico isquémico y que corresponde a tejido miocárdico infartado no viable (Figura 3$)^{29-31}$. Por el contrario, el edema miocárdico representa tejido miocárdico en riesgo, pero viable (Figura 3). Las posibles causas de IAM con arterias coronarias normales incluyen: la fisura de placa aterosclerótica con embolización distal, espasmo coronario, tromboembolismo coronario, disección coronaria espontánea, disfunción microvascular coronaria y puente miocárdico ${ }^{22,23,32-34}$. En 11 pacientes $(42,3 \%)$ se diagnosticó miocarditis, una enfermedad cardíaca aguda que puede simular un IAM al presentarse con dolor torácico opresivo acompañado de supradesnivel del segmento ST y elevación de la troponina plasmática. En estos pacientes, la RCM identifica edema miocárdico secundario al proceso inflamatorio existente que aumenta el contenido del agua celular y extracelular en el miocardio (Figura 2$)^{35-38}$ y realce tardío subepicárdico o intramural sin comprometer el endocardio, afectando con mayor frecuencia la pared lateral del VI (Figura 2) ${ }^{39-42}$. La localización del realce tardío en pacientes con sospecha de IAM con SDST y coronarias normales permite diferenciar entre IAM y miocarditis aguda ${ }^{40-41,43-45}$.
En ningún paciente con miocarditis se encontró alteraciones segmentarias de la contracción del VI en la ecocardiografía o cine-resonancia cardíaca. Una posible explicación de esta observación, sería la menor participación que el subepicardio tendría en la contracción miocárdica ${ }^{7}$ a diferencia de lo que ocurre en los pacientes con IAM y miocardiopatía de Takotsubo, en los cuales el daño es transmural o subendocárdico en los primeros, y transmural en los últimos. El diagnóstico de miocardiopatía de Takotsubo se confirmó en 6 pacientes $(23,1 \%)$, que es una enfermedad cardíaca aguda reversible que afecta principalmente a mujeres posmenopáusicas y que es habitualmente gatillada por un episodio de estrés emocional o físico agudo, antecedente presente en todas las pacientes en el presente estudio ${ }^{46-49}$. Cuatro pacientes desarrollaron una miocardiopatía de Takotsubo clásica con acinesia apical y circunferencial media del VI e hipercontractilidad de los segmentos basales como puede observarse en las Figuras 4 y $5^{49}$. Dos tenían una forma atípica con acinesia de los segmentos medio del VI e hipercontractilidad basal y apical (Figuras 4 y 5$)^{50,51}$. En estas pacientes, la RMC identificó edema miocárdico localizado en la zona discinética del VI con ausencia de realce tardío (Figura 4$)^{52}$, hallazgo que permite descartar IAM y miocarditis aguda ${ }^{53-55}$. La evolución de la mayoría de los pacientes con miocardiopatía de Takotsubo es buena, con regresión de las alteraciones segmentarias de la contracción y normalización de la función global del VI, tal como se comprobó en el control ecocardiográfico en nuestras pacientes (Figura 6). El mecanismo fisiopatológico para explicar el edema miocárdico en la miocardiopatía de Takotsubo se desconoce, pero podría derivar de isquemia transitoria y actividad inflamatoria descritos en esta enfermedad $^{52,56}$. La fracción de eyección del VI fue menor en los pacientes con miocardiopatía de Takotsubo en comparación a los pacientes con IAM y miocarditis aguda (ver tabla 2), y el valor máximo del péptido natriurético cerebral mayor respecto de los pacientes con IAM, observación concordante con reportes previos en la literatura ${ }^{57,59}$. La causa de la miocardiopatía de Takotsubo no se conoce aún, pero la explicación más plausible sería la toxicidad celular directa del cardiomiocito con aturdimiento miocárdico, producto de la elevada concentración plasmática de catecolaminas ${ }^{60,61}$. El vasoespasmo coronario es otra explicación 
arguible, aunque no se observa en la mayoría de los pacientes y cuando está presente, no se tiene claro si es un factor causal o un epifenómeno relacionado con los altos niveles de catecolaminas plasmáticas $^{62}$. El diagnóstico de miocardiopatía de Takotsubo se basa en los criterios recientemente propuestos por la Sociedad Europea de Cardiología ${ }^{48}$ en los que la RMC constituye una herramienta diagnóstica esencial. Su utilización permite diagnosticar IAM en aproximadamente un tercio de los pacientes hospitalizados con sospecha de miocardiopatía de Takotsubo ${ }^{63}$. Por otra parte, permite el diagnóstico diferencial entre un IAM y miocardiopatía de Takotsubo en pacientes con infarto de miocardio previo ${ }^{64}$.

La confirmación de un IAM en pacientes con arterias coronarias normales o el diagnóstico de miocarditis aguda o miocardiopatía de Takotsubo mediante RMC permite el tratamiento adecuado en cada caso cuyo beneficio en términos de seguridad y costos asociados es relevante ${ }^{65,66}$.

\section{Conclusiones}

La resonancia magnética cardiaca permite diagnosticar con exactitud el infarto agudo de miocardio en pacientes con arterias coronarias angiográficamente normales. Además, posibilita el diagnóstico diferencial de patologías que lo simulan como la miocardiopatía de Takotsubo y la miocarditis aguda.

\section{Referencias}

1. Codreanu A, Djaballah W, Angioi M, Ethevenot G, MoulinF, Felblinger J, et al. Detection of myocarditis by contrast enhanced MRI in patients presenting with acute coronary syndrome but no coronary stenosis. J Magn Reson Imaging 2007; 25 (5): 957-65.

2. Monney PA, Sekhri N, Burchell T, Knight C, Davies C, Deaner A, et al. Acute myocarditis presenting as acute coronary syndrome: role of early cardiac magnetic resonance in its diagnosis. Heart 2011; 97 (16): 1312-8.

3. Prasad A, Lerman A, Rihal CS. Apical ballooning syndrome [Tako-Tsubo or stress cardiomyopathy]: a mimic of acute myocardial infarction. Am Heart J 2008; 155 (3): 408-17.

4. Bybee KA, Kara T, Prasad A. Systematic review: transient left ventricular apical ballooning: a syndrome that mi- mics ST-segment elevation myocardial infarction. Ann Intern Med 2004; 141 (11): 858-65.

5. Thygesen K, Alpert JS, Jaffe AS, Chaitman BR, Bax JJ, Morrow DA, et al. Fourth universal definition of myocardial infarction. Eur Heart J 2019; 40 (3): 237-69.

6. Assomull RG, Lyne JC, Keenan N, Gulati A, Bunce NH, Davies SW, et al. The role of cardiovascular magnetic resonance in patients presenting with chest pain, raised troponin, and unobstructed coronary arteries. Eur Heart J 2007; 28 (10): 1242-9.

7. Laraudogoitia E, Pérez-David E, Larena JA, Velasco del Castillo S, Rumoroso JR, Onaindía JJ, et al. Utilidad de la resonancia magnética cardiaca en el diagnóstico de los pacientes con síndrome coronario agudo y coronarias normales. Rev Esp Cardiol 2009; 62 (9): 976-83.

8. Mahmoudi M, Harden S, Abid N, Peebles C, Nicholas $\mathrm{Z}$, Jones $\mathrm{T}$, et al. Troponin-positive chest pain with unobstructed coronary arteries: definitive differential diagnosis using cardiac MRI. Br J Radiol 2012; 85 (1016): e461-6.

9. Gallagher S, Jones DA, Mohiddin S. Diagnosis and management of patients with acute cardiac symptoms, troponin elevation and culprit-free angiograms. Heart 2012; 98 (13): 974-81.

10. Cerqueira MD, Weissman NJ, Dilsizian V, Jacobs AK, Kaul S, Laskey WK, et al. Standardized myocardial segmentation and nomenclature for tomographic imaging of the heart: a statement for healthcare professionals from the Cardiac Imaging Committee of the Council on Clinical Cardiology of the American Heart Association. Circulation 2002; 105 (4): 539-42.

11. Eitel I, Friedrich MG. T2-weighted cardiovascular magnetic resonance in acute cardiac disease. J Cardiovasc Magn R 2011; 13 (1): 13.

12. Doltra A, Amundsen BH, Gebker R, Fleck E, Kelle S. Emerging concepts for myocardial late gadolinium enhancement MRI. Curr Cardiol Rev 2013; 9 (3): 185-90.

13. Doltra A, Stawowy P, Dietrich T, Schneeweis C, Fleck E, Kelle S. Magnetic Resonance Imaging of Cardiovascular Fibrosis and Inflammation: From Clinical Practice to Animal Studies and Back. BioMed Res Int 2013; 1-10.

14. Gu YL, Svilaas T, van der Horst IC. Conditions mimicking acute ST-segment elevation myocardial infarction in patients referred for primary percutaneous coronary intervention. Neth Heart J 2008; 16 (10): 325-31.

15. Groot HE, Wieringa WG, Mahmoud KD, Lexis CP, Hiemstra B, van der Harst P, et al. Characteristics of patients with false- ST-segment elevation myocardial infarction diagnoses. Eur Heart J Acute Cardiovasc Care 2016; 5 (4): 339-46.

16. Nfor T, Kostopoulos L, Hashim H, Jan MF, Gupta A, 
Bajwa $\mathrm{T}$, et al. Identifying false positive ST-elevation myocardial infarction in emergency department patients. J Emerg Med 2012; 43 (4): 561-7.

17. Larson DM, Menssen KM, Sharkey SW, Duval S, Schwartz RS, Harris J, et al. "False-positive" cardiac catheterization laboratory activation among patients with suspected ST-segment elevation myocardial infarction. JAMA 2007; 298 (23): 2754-60.

18. Friedrich MG, Abdel-Aty H, Taylor A, Schulz-Menger J, Messroghli D, Dietz R. The salvaged area at risk in reperfused acute myocardial infarction as visualized by cardiovascular magnetic resonance. J Am Coll Cardiol 2008; 51 (16): 1581-17.

19. Wright J, Adriaenssens T, Dymarkowski S, Desmet W, Bogaert J. Quantification of myocardial area at risk with T2-weighted CMR: comparison with contrast-enhanced CMR and coronary angiography. JACC Cardiov Imaging 2009; 2 (7): 825-31.

20. Dastidar AG, Rodrigues JCL, Johnson TW, De Garate E, Singhal P, Baritussio, et al. Myocardial Infarction with Nonobstructed Coronary Arteries. JACC Cardiov Imaging 2017;10(10):1204-6.

21. Ibanez B, James S, Agewall S, Antunes MJ, Bucciarelli-Ducci C, Bueno H, et al. 2017 ESC Guidelines for the management of acute myocardial infarction in patients presenting with ST-segment elevation. Eur Heart J 2018; 39 (2): 119-77.

22. Tamis-Holland J E, Jneid H, Reynolds HR, Agewall S, Brilakis ES, Brown TM. Contemporary Diagnosis and Management of Patients with Myocardial Infarction in the Absence of Obstructive Coronary Artery Disease: A Scientific Statement from the American Heart Association. Circulation 2019; 139 (18): e891-e908.

23. Gue YX, Kanji R, Gati S, Gorog DA. MI with Non-obstructive Coronary Artery Presenting with STEMI: A Review of Incidence, Aetiology, Assessment and Treatment. Eur Cardiol 2020;15: e20.

24. Pustjens TFS, Appelman Y, Damman P, et al. Guidelines for the management of myocardial infarction/injury with non-obstructive coronary arteries (MINOCA): a position paper from the Dutch ACS working group [published correction appears in Neth Heart J 2020 Jan;28(1):59]. Neth Heart J 2020;28(3):116-130.

25. Berry C, Kellman P, Mancini C, Chen MY, Bandettini WP, Lowrey $\mathrm{T}$, et al. Magnetic resonance imaging delineates the ischemic area at risk and myocardial salvage in patients with acute myocardial infarction. Circ Cardiovasc Imaging 2010; 3(5): 527-35.

26. Schwitter J, Arai AE. Assessment of cardiac ischaemia and viability: role of cardiovascular magnetic resonance. Eur Heart J 2011; 32 (7): 799-809.
27. Kim HW, Farzaneh-Far A, Kim RJ. Cardiovascular magnetic resonance in patients with myocardial infarction: current and emerging applications. J Am Coll Cardiol 2009; 55 (1): 1-16.

28. Wong RY, Farzaneh-Far A. Measuring myocardial scar by CMR. JACC Cardiov Imaging 2011;4(2):157-160.

29. Ahmed N, Carrick D, Layland J, Oldroyd KG, Berry C. The role of cardiac magnetic resonance imaging (MRI) in acute myocardial infarction (AMI). Heart Lung Circ 2013; 22 (4): 243-55.

30. Masci PG, BogaertJ. Post myocardial infarction of the left ventricle: The course ahead seen by cardiac MRI. Cardiovasc Diagn Ther 2012; 2 (2): 113-27.

31. Rajiah P, Desai MY, Kwon D, Flamm SD. Imaging of Myocardial Infarction. RadioGraphics 2013; 33 (5): 1383-412.

32. Niccoli G, Scalone G, Crea F. Acute myocardial infraction with no obstructive coronary atherosclerosis: mechanisms and management. Eur Heart J 2015; 36 (8): 475-81.

33. Agewall S, Beltrame JF, Reynolds HR, Niessner A, Rosano $\mathrm{G}$, Caforio AL, et al. ESC working group position paper on myocardial infarction with non-obstructive coronary arteries. Eur Heart J 2017; 38 (3): 143-53.

34. Tamis-Holland J, Jneid H, Reynolds HR, Agewall S, Brilakis ES, Brown TM, et al. Contemporary diagnosis and management of patients with myocardial infarction in the abcense of obstructive coronary artery disease. Circulation 2019; 139 (18): e891-e908.

35. Abdel-Aty H, Zagroseck A, Wassmuth R, Kumar A, Messoroghli D, Bock P, et al. Diagnostic performance of cardiovascular magnetic resonance in patients with suspected acute myocarditis: comparison of different approaches. J Am Coll Cardiol 2005; 45 (11): 1815-22.

36. Zagroseck A, Wassmuth R, Abdel-Aty H, Rudolph A, Dietz, Schulz-Menger J. Relation between myocardial edema and myocardial mass during the acute and convalescent phase of myocarditis - a CMR study. J Cardiovasc Magn R 2008; 10 (1): 19.

37. Friedrich MG, Marcotte F. Cardiac magnetic resonance assessment of myocarditis. Circ Cardiovasc Imag 2013; 6 (5): 833-9.

38. Yilmaz A, Ferreira V, Klingel K, Kandolf R, Neubauer S, Sechtem U. Role of cardiovascular magnetic resonance imaging (CMR) in the diagnosis of acute and chronic myocarditis. Heart Fail Rev 2013; 18 (6): 747-60.

39. Skouri HN, Dec GW, Friedrich MG, Cooper LT. Noninvasive imaging in miocarditis. J Am Coll Cardiol 2006; 48 (10): 2085-93.

40. Mahrholdt H, Wagner A, Judd RM, Sechtem U, Kim RJ. Delayed enhancement cardiovascular magnetic reso- 
nance assessment of non-ischaemic cardiomyopathies. Eur Heart J 2005; 26 (15): 1461-74.

41. Mahrholdt H, Wagner A, Dluigi CC, Kispert E, Hager S, Meinhardt G, et al, et al. Presentation, patterns of myocardial damage, and clinical course of viral myocarditis. Circulation 2006; 114 (15): 1581-90.

42. Alsaileek A, Nasim M, Aljizeeri A, Alharthi M, Al-Mallah MH. The role of delayed contrast-enhanced cardiac magnetic resonance in differentiating myocarditis from myocardial infarction. Eur Heart J 2014; 16 (suppl_B): B24-8.

43. Friedrich MG, Sechtem U, Schulz-Menger J, Holmvang G, Alakija P, Cooper LT, et al. Cardiovascular Magnetic Resonance in Myocarditis: A JACC White Paper. J Am Coll Cardiol 2009; 53 (17): 1475-87.

44. Pozo E and Sanz J. Differentiating infarction from myocarditis. Heart Metab 2014; 62: 13-7.

45. Tornvall P, Gerbaud E, Behaghel A, Chopard R, Collste O, Laraudogoitia E, et al. Myocarditis or "true" infarction by cardiac magnetic resonance in patients with a clinical diagnosis of myocardial infarction without obstructive coronary disease: A meta-analysis of individual patient data. Atherosclerosis 2015; 241 (1): 87-91.

46. Gianni M, Dentali F, Grandi AM, Sumner G, Hiralal R, Lonn E. Apical ballooning syndrome or Takotsubo cardiomyoptahy: a systematic review. Eur Heart J 2006; 27 (13): 1523-9.

47. Agarwal S, Bean, MG, Hata, JS, Castresana, MR. Perioperative takotsubo cardiomyopathy: a systematic review of published cases. Semin Cardiothorac Vasc Anesth 2017; 21 (4): 277-90.

48. Lyon AR, Bossone E, Schneider B, Sechtem U, Underwood SR, Sheppard MN, et al. Current state of knowledge on Takotsubo syndrome: a Position Statement from the Taskforce on Takotsubo Syndrome of the Heart Failure Association of the European Society of Cardiology. Eur J Heart Fail 2016; 18 (1): 8-2.

49. Ghadri JR, Wittstein IS, Prasad A, Sharkey S, Dote K, Akashi YJ, et al. International Expert Consensus Document on Takotsubo Syndrome (Part I): Clinical Characteristics, Diagnostic Criteria, and Pathophysiology. Eur Heart J 2018; 39 (22): 2032-46.

50. Haghi D, Papavassiliu T, Fluchter S, Kaden JJ, Porner T, Borggrefe M, et al. Variant form of the acute apical ballooning syndrome (takotsubo cardiomyopathy): observations on a novel entity. Heart 2006; 92 (3): 392-54.

51. Templin C, Ghadri JR, Diekmann J, Napp LC, Bataiosu DR, Jaguszwski M, et al. Clinical Features and Outcomes of Takotsubo (Stress) Cardiomyopathy. New Engl J Med 2015;373(10):929-938.

52. Eitel I, Lucke C, Grothoff M, Sareban M, Schuler Get al.
Inflammation in Takotsubo cardiomyopathy: insights from cardiovascular magnetic resonance imaging. Eur Radiol 2010; 20 (2): 422-31.

53. Teraoka K, Kiuchi S, Takada N, Hirano M, Yamashina A. Images in cardiovascular medicine. No delayed enhancement on contrast magnetic resonance imaging with Takotsubo cardiomyopathy. Circulation 2005; 111 (16): e261-2.

54. Mitchell JH, Hadden TB, Wilson JM, Achari A, Muthupillai R, Flamm SD. Clinical features and usefulness of cardiac magnetic resonance imaging in assessing myocardial viability and prognosis in takotsubo cardiomyopathy (transient left ventricular apical ballooning syndrome). Am J Cardiol 2007; 100 (2): 296-301.

55. Cacciotti L, Passaseo I, Marazzi G, Camastra G, Campolongo G, Beni S, et al G. Observational study on Takotsubo-like cardiomyopathy: clinical features, diagnosis, prognosis and follow-up. BMJ Open 2012; 2 (5): e001165.

56. Bratis K. Cardiac Magnetic Resonance in Takotsubo Syndrome. Eur Cardiol 2017; 12 (1): 58-62.

57. Guadri JR, Wittstein IS, Prasad A, Sharkey S, Dote K, Akashi YJ, et al. International Expert Consensus Document on Takotsubo Syndrome (Part II): Diagnostic Workup, Outcome, and Management. Eur Heart J 2018; 39 (22): 2047-62.

58. Grabowski M, Filipiak KJ, Malek LA, Piatkowski R, Scislo P, Karpinski G, et al. Increased B-type natriuretic peptide levels in patients with apical ballooning syndrome - consecutive cases report. Int J Cardiol 2008; 124 (3): 404-6.

59. Madhavan M, Borlaug BA, Rihal CS, Prasad A. Stress hormone and circulating biomarker profile of apical ballooning syndrome (Takotsubo cardiomyopathy): insights into the clinical significance of B-type natriuretic peptide and troponin levels. Heart 2009; 95 (17): 1436-41.

60. Akashi Y, Nef H, Lyon A. Epidemiology and pathophysiology of Takotsubo syndrome. Nat Rev Cardiol 2015; 12 (7): 387-97.

61. Pellicia F, Kaski JC, Crea F, Camici PG. Pathophysiology of Takotsubo Syndrome. Circulation 2017; 135 (24): 2426-41.

62. Kato K, Lyon AR, Ghadri JR, Templin C. Takotsubo syndrome: aetiology, presentation and treatment. Heart 2017;103(18):1461-1469.

63. Gandhi H, Rodriguez JE, Reynolds H. Takotsubo Cardiomyopathy versus Apical Infarction in patients with Myocardial infarction and non-obstructive coronary artery disease (MINOCA). J Am Coll Cardiol 2017; 69 (11): 270 . 
64. Díaz-Navarro R, Villagran F. Takotsubo cardiomyopathy and coronary artery disease: value of cardiac magnetic resonance imaging for diagnostic confirmation: a case report. Eur Heart J Case Rep 2018; 3 (1): yty151.

65. Hausvater A, Pasupathy S, Tornvall P, Gandhi H, Tavella R, Beltrame J, et al. ST-segment elevation and cardiac magnetic resonance imaging findings in myocardial infarction with non-obstructive coronary arteries. Int J Cardiol 2019; 287: 128-31.

66. Vágó H, Szabó L, Dohy Z, Czimbalmos C, Tóth A, Ferenc $S$, et al. Early cardiac magnetic resonance imaging in troponin-positive acute chest pain and non-obstructed coronary arteries. Heart 2020; 106 (13): 992-1000. 